\title{
Activation of the sweet taste receptor T1R3 by sucralose attenuates VEGF-induced vasculogenesis in a cell model of the retinal microvascular endothelium
}

\author{
Polina Lizunkova ${ }^{1} \cdot$ Emmanuella Enuwosa $^{1} \cdot$ Havovi Chichger ${ }^{1}$
}

Received: 8 May 2018 / Revised: 20 September 2018 / Accepted: 3 October 2018 / Published online: 23 October 2018

(C) The Author(s) 2018

\begin{abstract}
Background One of the most prevalent microvascular complications for patients with diabetes is diabetic retinopathy (DR) associated with increased retinal endothelial blood vessel formation. Treatments to reduce vascularisation in the retinal endothelium are linked to improved sight in patients with DR. Recently, we have demonstrated the novel protective role of the artificial sweetener, sucralose, and the sweet taste receptor, T1R3, in the pulmonary endothelium to reduce vascular leak. In the present study, we examined the role of sucralose and sweet taste receptors on vasculogenic processes (proliferation, migration, adhesion and tube formation) in a cell model of the retinal endothelium.

Methods We exposed human retinal microvascular endothelial cells (RMVEC) to VEGF as an in vitro model of DR in the presence and absence of T1R3 agonist sucralose.

Results In RMVEC, we observed increased VEGF-induced cell proliferation, migration, adhesion and tube formation, which was significantly attenuated by exposure to the artificial sweetener sucralose. Following siRNA knockdown of the sweet taste receptor, T1R3, but not T1R2, the protective effect of sucralose on VEGF-induced RMVEC vasculogenic processes was blocked. We further demonstrate that sucralose attenuates VEGF-induced Akt phosphorylation to protect the retinal microvasculature.

Conclusion These studies are the first to demonstrate a protective effect of an artificial sweetener, through the sweet taste receptor T1R3, on VEGF-induced vasculogenesis in a retinal microvascular endothelial cell line.
\end{abstract}

Keywords Vasculogenesis $\cdot$ Diabetic retinopathy $\cdot$ Sweet taste receptors $\cdot$ Artificial sweeteners $\cdot$ Endothelium

\section{Introduction}

Worldwide, there are currently 425 million people estimated to suffer from type 1 and 2 diabetes, which results in a yearly healthcare expenditure of 727 billion USD. By 2045, the number of sufferers is predicted to increase to 693 million, which will further increase the economic burden of the disease [1]. One of the most common and debilitating complications associated with diabetes is the development of diabetic eye

Electronic supplementary material The online version of this article (https://doi.org/10.1007/s00417-018-4157-8) contains supplementary material, which is available to authorized users.

Havovi Chichger

Havovi.Chichger@anglia.ac.uk

1 Biomedical Research Group, Department of Biomedical and Forensic Sciences, Anglia Ruskin University, East Road, Cambridge CB1 1PT, UK disease associated with diabetic retinopathy (DR), diabetic macular edema and glaucoma [1]. Over $90 \%$ of patients with type 1 diabetes and around $60 \%$ of patients with type 2 diabetes will suffer from a level of DR ranging from blurred vision to complete vision loss [2]. Given the increasing incidence of diabetes, there is thus a significant need to understand the mechanisms which regulate DR, with the aim of developing effective therapeutic agents for the complication.

Endothelial cell injury is a hallmark of the microvascular complications observed due to chronic hyperglycaemia in diabetes. Indeed, small improvements in glycosylatedhaemoglobin directly correlate with a significantly reduced risk of developing DR [3]. Hyperglycaemia increases metabolic disruption, inflammation and hypoxia in patients with diabetes, associated with the pathophysiology of DR [2]. DR, characterised by retinal lesions, results in morphological changes and increases in vascular permeability of the retinal microvasculature [4]. In non-proliferative retinopathy, this permeability causes macular edema which, over time, leads 
to capillary widening and degeneration of the retina whereas in proliferative retinopathy, there is excessive formation of blood vessels (vascularisation) around the optic disk; this aberrant neovascularisation is a major contributor to vision loss and has been attributed to hyperglycaemia-induced increase in local vitreous vascular endothelial growth factor (VEGF) levels in patients with diabetes [5-8]. Indeed, current treatment for patients with non-proliferative DR utilises antiVEGF agents to reduce macular edema and improve outcomes for patients $[4,9]$. However, there are concerns regarding the safety of anti-VEGF agents over the long term, with a small number of cases indicating side-effects such as an increased risk of neurodegeneration for the remaining healthy retina $[10,11]$. There is thus a need to investigate potential alternatives to current anti-VEGF agents to improve outcomes for patients with DR.

We have previously demonstrated a role for the commonlyconsumed artificial sweetener, sucralose, in attenuating VEGF-induced vascular leak in the pulmonary endothelium [12]. These studies indicate that, through binding to the sweet taste receptor T1R3, sucralose tightens the microvascular endothelium and protects the barrier against leak from several agonists including the bacterial endotoxin LPS. Whilst these studies indicate a protective effect of the sweet taste sensing pathway in reducing leak at the pulmonary microvasculature, a role for artificial sweeteners in regulating the retinal endothelium has not been previously studied.

Sweet taste sensing is mediated by the sweet taste receptors T1R2 and T1R3 which form a heterodimer; however, T1R3 is also able to form a homodimer [13]. These G protein coupled receptors (GPCRs) are stimulated by low concentrations of acutely sweet molecules such as artificial sweeteners $(<$ $1 \mathrm{mM}$ ) or exceedingly high concentrations of glucose (> $300 \mathrm{mM}$ ) [14]. We previously demonstrated that sucralose regulates expression and phosphorylation of key signalling molecules such as p110 $\alpha$-PI3K, MLC2 and Src in the pulmonary endothelium [12]. Interestingly, these signalling molecules are all linked to processes associated with neovascularisation, including endothelial cell migration, adhesion, contraction and tube formation [15-17]. Therefore, we hypothesised that T1R2 and T1R3 signalling, activated by sucralose, would have an effect on vasculogenic potential of the retinal endothelium.

In the present study we investigate, for the first time, the role of the sweet taste receptor in regulating the retinal endothelium. We demonstrate that activation of the sweet taste receptor, with the artificial sweetener sucralose, attenuates VEGF-induced leak across the retinal endothelial barrier. We further demonstrate that VEGF mediates excessive cell migration, adhesion, proliferation and tube formation in a retinal microvascular endothelial cell line which is blocked by sucralose. Interestingly, the protective effect of sucralose is mediated through the sweet taste receptor T1R3 but not
T1R2. Finally, we demonstrate a role for Akt as a key signalling molecule downstream of T1R3 which regulates the protective effect of sucralose. Our studies show that sweet taste sensing through T1R3 plays a significant role in aberrant vascularisation processes which are seen in diabetes and highlights a potential anti-VEGF therapeutic agent for patients with DR.

\section{Methods}

\section{Cell lines, reagents and ethics}

Human retina (RMVEC) and lung (LMVEC) microvascular endothelial cells were purchased from Cell Systems (Kirkland, WA) and ATCC (Teddington, UK) respectively. RMVEC and LMVEC were cultured in vascular cell media supplemented with culture boost or endothelial cell BBE kit, respectively. Endothelial cells were used between passage 2 and 9 and maintained the traditional endothelial cell characteristics of von Willebrand factor and vascular endothelial (VE)-cadherin expression, uptake of acetylated LDL, and positive staining for the lectin Griffonia simplicifolia.

TRIzol, SuperscriptII and recombinant human VEGF protein were purchased from ThermoFisher (Paisley, UK). siRNA and DharmaFECT TM reagent were obtained from Dharmacon (Cambridge, UK). Antibodies directed against T1R2, T1R3, VEGFR2 (Flk-1), phosphorylated $\left(\mathrm{Ser}^{473}\right)$ and total Akt1/2, and actin were purchased from Santa Cruz Biotechnology (Santa Cruz, CA). Antibodies were selected from validation studies (https://scicrunch.org/resources). Matrigel Basement Membrane Matrix was obtained from BD Biosciences (Oxford, UK). All other reagents were purchased from Sigma Aldrich (Dorset, UK).

All experimental protocols were approved by the Departmental Research Ethics Panel at Anglia Ruskin University prior to start.

\section{Transient transfection}

RMVEC were transiently transfected with T1R2 or T1R3 SMARTpool siGENOME siRNA duplexes $(300 \mathrm{nM})$, or non-specific, scrambled duplexes, using the Dharmafect ${ }^{\mathrm{TM}}$ reagent 4 , as per the manufacturer's guidelines. At $42 \mathrm{~h}$ following transfection, cells were exposed to sucralose $(0.1 \mathrm{mM})$ in the presence or absence of zinc sulphate $(0.7 \mathrm{mM})$, VEGF $(100 \mathrm{ng} / \mathrm{ml})$ or SC79 $(10 \mu \mathrm{M})$ for $6 \mathrm{~h} . \mathrm{H}_{2} \mathrm{O}$ was used as a vehicle for all treatments except $\mathrm{SC} 79$, where ethanol was used. Confirmation of knockdown was performed using RTPCR and Western blotting analysis of mRNA and protein expression respectively. 


\section{RT-PCR}

Total RNAs were extracted from LMVEC and RMVEC using the TRIzol reagent as per the manufacturer's instructions. RNA was purified using the acid phenol/chloroform system and reverse transcribed using SuperScriptII. T1R2 and T1R3 transcripts were measured with human $\beta$-actin primers (forward primer CACCAACTGGGACGACAT; reverse primer ACAGCCTGGATAGCAACG) used as the house-keeping gene as described previously [18]. Expression of the Tas $1 r 2$ and Tas $1 r 3$ gene was measured using specific intron-spanning primers (T1R2: forward primer AATGTCCAGCCGGT GCTCTA, reverse primer CATCGCTGATGGCGCTGTA; T1R3: forward primer TTCCCCCAGTACGTGAAGAC, reverse primer CAGAGAACGTCTGGTGGTGA). Relative gene expression level was analysed, for each sample, using the $\Delta \mathrm{Ct}$ method where $\Delta \mathrm{Ct}=\left(\mathrm{Ct}_{\text {Tas1r }}-\mathrm{Ct}_{\beta \text {-actin }}\right)$ corresponding to the detected threshold cycles for the target gene and $\beta$-actin control.

\section{Western blotting}

LMVEC and RMVEC were lysed with RIPA buffer, resuspended in Laemmli buffer $(50 \mu \mathrm{g})$ and subjected to immunoblot analysis. Immunoblot analyses were performed on $10 \%$ SDS-PAGE using primary antibodies specific to T1R2, T1R3, phosphorylated Akt1/2 $\left(\mathrm{Akt}^{\mathrm{S} 473}\right)$, total $\mathrm{Akt1} / 2$ and $\beta$-actin at a dilution of 1:1000, except actin (1:5000), and secondary antibody at dilutions of 1:5000. Densitometry was performed using gel analysis software on ImageJ.

\section{Whole cell ELISA}

RMVEC were transiently transfected with siRNA for $42 \mathrm{~h}$, followed by exposure to sucralose and VEGF for a further $6 \mathrm{~h}$. Cells were then rinsed once with DPBS and fixed using $1 \%$ paraformaldehyde at room temperature for $10 \mathrm{~min}$. Whole cell ELISA was then performed as previously described $[12,19]$ using antibodies specific to the extracellular domain of T1R2 (H-90, $\alpha \alpha$ 201-390), T1R3 (G-2, $\alpha \propto$ 320-499) and VEGFR2 (Flk-1, Q-20, $\alpha \alpha$ unspecified), and fluorescent-conjugated secondary antibodies measured at $1 \mathrm{~s}$ exposure time using a florescent plate reader (Victor, Perkin Elmer).

\section{Endothelial monolayer permeability}

Endothelial monolayer permeability was assessed using the FITC-dextran permeability assay and validated with TER $\left(\mathrm{EVOM}^{2}\right.$; World Precision Instruments, Herts, UK). For analysis of monolayer permeability, RMVEC were transiently transfected with siRNA for $42 \mathrm{~h}$ on collagen-coated Transwell filters followed by exposure to sucralose, VEGF and zinc sulphate. Addition of treatments was made at the same time and permeability was measured at $6 \mathrm{~h}$ following treatment. FITC-conjugated to $40 \mathrm{kDa}$ dextran was added to media in the upper chamber of the Transwell filter, allowed to equilibrate for $360 \mathrm{~s}$ at $37^{\circ} \mathrm{C}$, and a sample $(100 \mu \mathrm{l})$ of media from the lower chamber was collected and analysed at $488 \mathrm{~nm}$ using a fluorescent plate reader (Victor, Perkin Elmer). Permeability (\%) was calculated by fluorescence accumulated in the lower chamber divided by fluorescence in the upper chamber, multiplied by 100 .

\section{Cell viability assay}

RMVEC were transiently transfected with siRNA for $42 \mathrm{~h}$, followed by exposure to sucralose for a further $6 \mathrm{~h}$. Viability was assessed using the Cell Counting Kit-8 (CCK-8) as per the manufacturer's guidelines with absorbance read at $450 \mathrm{~nm}$ using a microplate reader (Tecan Sunrise). Viability was calculated as $\%$ normalised to vehicle.

\section{Cell proliferation assay}

RMVEC were transiently transfected with siRNA for $24 \mathrm{~h}$, and then quiesced in media with $1 \%$ FBS for a further $18 \mathrm{~h}$. Cells were exposed to sucralose, VEGF, zinc sulphate and SC79, prepared in media with $1 \% \mathrm{FBS}$, for $6 \mathrm{~h}$ and counted using a haemocytometer.

\section{Cell migration assay}

RMVEC were transiently transfected with siRNA for $42 \mathrm{~h}$, and scratched using a pipette tip and immediately treated with sucralose, VEGF, zinc sulphate and SC79 for a further $6 \mathrm{~h}$. Cell migration was monitored at $2 \mathrm{~h}$ time intervals following the initial scratch and images were captured at $\times 10$ magnification using a Zoe ${ }^{\mathrm{TM}}$ Cell Imager (BioRad). Cell migration was assessed using the MiToBo analyser software in Image J as previously described [20], with an average was assessed from 2 wells to represent an $n$ of 1 .

\section{Cell adhesion assay}

RMVEC were transiently transfected with siRNA for $46 \mathrm{~h}$, and then replated and immediately exposed to sucralose, VEGF, zinc sulphate and SC79 for a further $2 \mathrm{~h}$. Cells were then rinsed once with DPBS and the CCK-8 kit was used (as described in 'Cell viability assay') to quantify adherent cells.

\section{In vitro tube formation}

Transfected RMVEC were plated directly onto Matrigel ${ }^{\mathrm{TM}}$ coated wells for $42 \mathrm{~h}$ at $37{ }^{\circ} \mathrm{C}$. Cells were then exposed to sucralose, VEGF, zinc sulphate and SC79 for a further $6 \mathrm{~h}$. Images of tube formation were captured at $\times 10$ magnification using a Zoe ${ }^{\mathrm{TM}}$ Cell Imager (BioRad). The number of joints 
and tubes were calculated by using the Angiogenesis Analyser software in Image $\mathbf{J}$ as previously described [20]. An average from two wells was assessed to represent an $n$ of 1 .

\section{Statistical analysis}

The experimental number is presented in the legend for each experiment. For two groups, the variance in data sets was analysed using the Mann-Whitney test followed by the appropriate $t$ test. For three or more groups, variance was assessed by using Bartlett's test with data sets not reaching significance studied by Kruskal-Wallis test followed by Dunn's test. For all other data sets, differences among the means were tested for significance in all experiments by ANOVA with Tukey's range significance difference test. Significance was reached when $p<0.05$. Values are presented as mean \pm standard error mean (S.E.M.)

\section{Results}

\section{Expression of the sweet taste receptors, T1R2 and T1R3, in a human retinal microvascular endothelial cell line}

The sweet taste receptors, T1R2 and T1R3, form a heterodimeric complex (T1R2/T1R3) or T1R3 forms a homodimeric complex (T1R3/T1R3) for sweet taste sensing [13]. We have a (i)

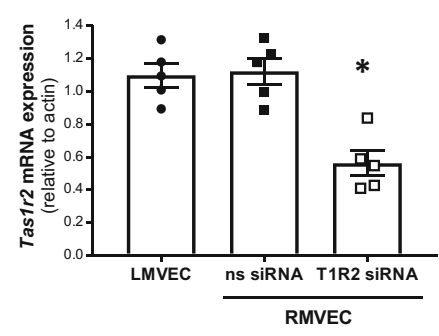

c

(i)

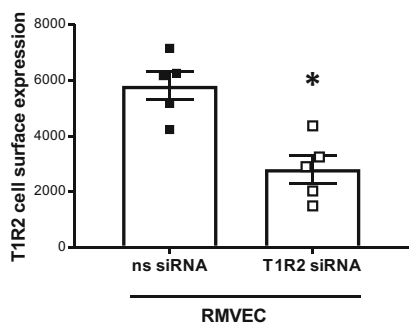

(ii)
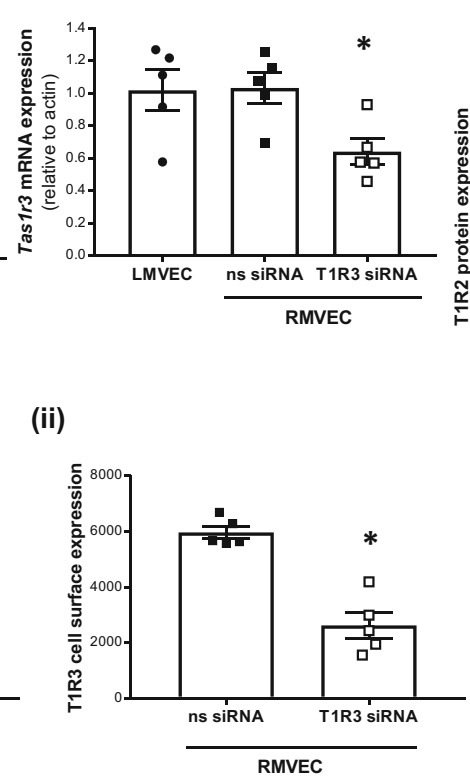

Fig. 1 Expression of the sweet taste receptors, T1R2 and T1R3, in retinal microvascular endothelial cells. mRNA (panel $a$ ) and protein expression (panel $b$ and $c$ ) of the T1R2 (i) and T1R3 (ii) gene and protein in retinal microvascular endothelial cells (RMVEC) and positive control lung microvascular endothelial cells (LMVEC). siRNA knockdown of T1R2 and T1R3 was performed in RMVEC to validate expression. Gene and previously demonstrated mRNA and protein expression of T1R3 in the pulmonary endothelium, at levels similar to that of the small intestine [12]. Here we demonstrate that mRNA and protein expression levels of both T1R2 and T1R3 in RMVEC were comparable to expression in pulmonary endothelial cells (LMVEC) (Fig. 1a, b). T1R2 and T1R3 are G protein coupled receptors which function at the plasma membrane [13] therefore we next assessed localisation of the receptors to the cell surface. Using extracellular antibodies and whole cell ELISA, expression of both T1R2 and T1R3 was demonstrated at the cell surface of RMVEC (Fig. 1c). To confirm specificity of primers and antibodies, siRNA knockdown of each receptor was performed. The expression of mRNA, whole cell and cell surface protein was significantly reduced, to a similar degree, following knockdown of T1R2 and T1R3 (Fig. 1a-c). These studies demonstrate, for the first time, that the sweet taste receptors, T1R2 and T1R3, are expressed in a human retinal microvascular endothelial cell model.

\section{Artificial sweetener sucralose attenuates VEGF-induced vasculogenic processes in a retinal microvascular endothelial cell model}

We next sought to establish whether activation of T1R2 and T1R3, by low concentrations of acutely sweet molecules [21], exhibited an effect on retinal endothelial cell function. As sucralose did not exert any effect on RMVEC viability

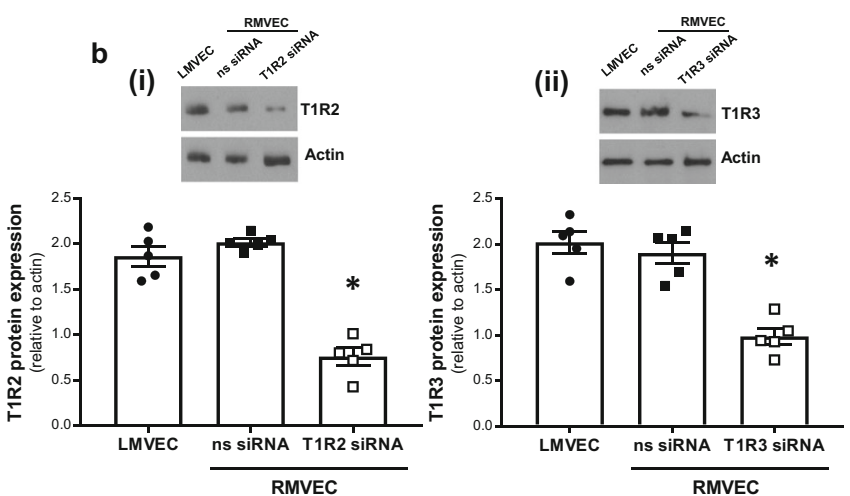

protein expression is analysed relative to $\beta$-actin. A representative blot is included for Western blotting (inset, panel b). Cell surface expression (panel c) of T1R2 (i) and T1R3 (ii) was determined with whole cell ELISA in retinal microvascular endothelial cells. $n=5$. Data is expressed as mean \pm S.E.M. $* p<0.05$ versus ns siRNA 
(Fig. 2a), the artificial sweetener was utilised at the concentration previously identified to be protective against endotoxininduced barrier disruption in the pulmonary endothelium $(0.1 \mathrm{mM})$ [12]. We next sought to establish whether sucralose was protective against VEGF-induced permeability in the cell model of the retinal endothelium. Exposure of RMVEC to VEGF significantly decreased monolayer resistance and increased permeability as measured by TER and FITC-dextran permeability assay respectively (Fig. 2b); however, VEGFinduced permeability was significantly attenuated by sucralose (Fig. 2b (ii)). Whilst permeability is an indicator of nonproliferative DR, a key pathophysiology of proliferative DR is aberrant vasculogenesis of the retinal microvasculature [6]. Therefore, we next studied the effect of sucralose on vasculogenic processes in the retinal endothelium. RMVEC exposed to sucralose alone, in the absence of VEGF, exhibited no change in cell proliferation, adhesion, migration or tube formation (Fig. 2c-f). RMVEC exposed to VEGF displayed a significant increase in proliferation, adhesion and migration, which was blocked by sucralose (Fig. 2c-e). Likewise, VEGF-induced tube formation and angiogenic potential, denoted as number of joints, in RMVEC was significantly attenuated by exposure to sucralose (Fig. 2f). These studies demonstrate that activation of the sweet taste receptors, T1R2 and T1R3, exerts a protective effect on the retinal endothelial cell model, to blunt VEGF-induced vasculogenic processes. Similar to VEGF, exposure of the endothelium to high glucose concentrations has been demonstrated to increase vasculogenic processes in the endothelium, linked to DR [22]. Exposure of RMVEC to sucralose significantly reduced high glucose-induced cell migration and proliferation (data not shown), indicating a pan-protective role of sucralose on the retinal endothelium.

\section{Sucralose attenuates VEGF-induced vasculogenic processes in retinal microvascular endothelial cells through the sweet taste receptor T1R3}

To study whether sucralose acts on retinal endothelial cells through the sweet taste receptors, T1R2 and T1R3, the next experiments were performed with inhibition of the sweet taste receptors. Zinc sulphate, a chemical inhibitor of sweet taste receptor activity, with no impact on sweet taste receptor whole cell or cell surface expression (Fig. 3a, b) was a

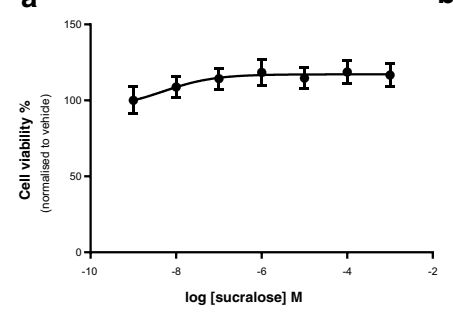

d

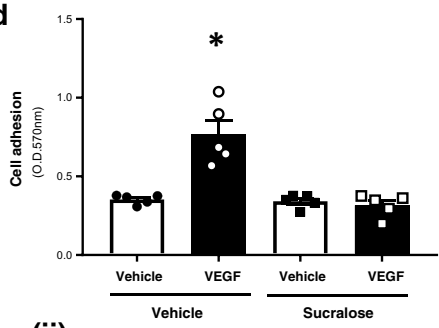

(ii)

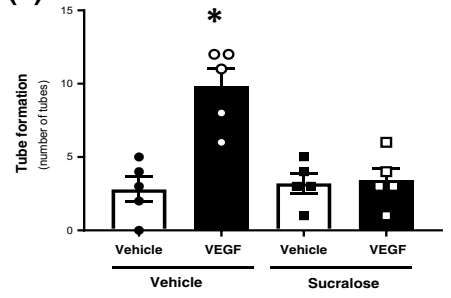

(i)

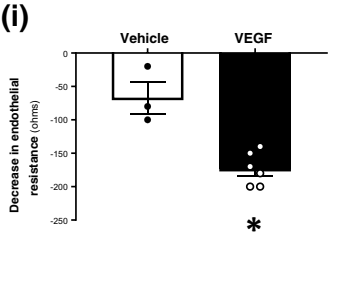

e

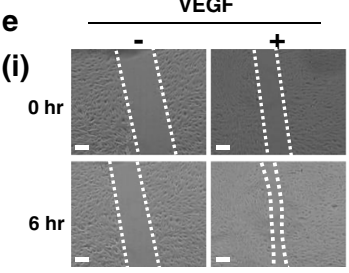

(iii)

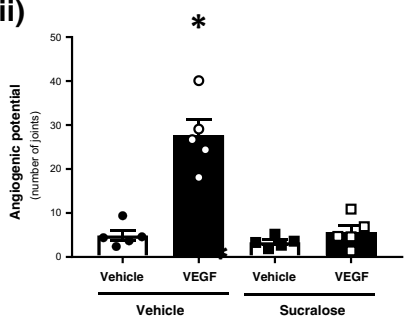

(ii)
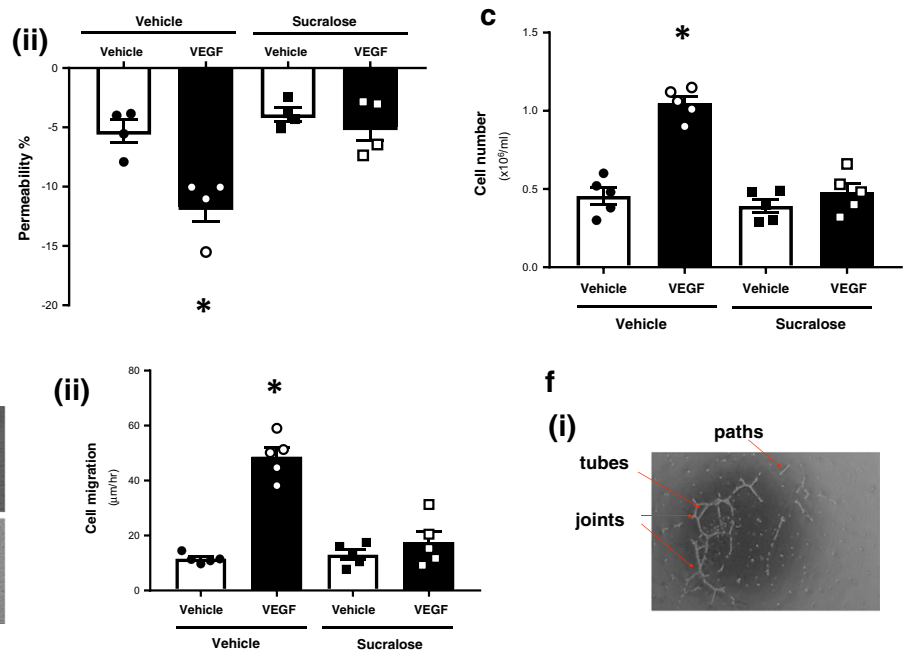

f

(i)

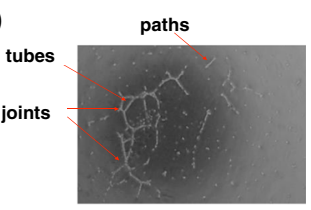

Fig. 2 An agonist for sweet taste receptors T1R2 and T1R3, sucralose, attenuates VEGF-induced angiogenic processes in the retinal microvascular endothelium. Panel $a$ : cell viability of RMVEC was measured by CCK8 assay following exposure to sucralose (1 nM$1 \mathrm{mM})$. Panel $b-f$ : changes in retinal endothelial cell: monolayer permeability (panel $b$ ), proliferation (panel $c$ ), adhesion (panel $d$ ), migration (panel e), tube formation (panel $f(i i)$ ) and angiogenic potential (panel $f($ iii)) were measured following exposure to sucralose $(0.1 \mathrm{mM})$ in the presence (closed bars) and absence (open bars) of VEGF $(100 \mathrm{ng} / \mathrm{ml})$. Validation of FITC-dextran permeability assay is shown using TER with VEGF (panel $b(i)$ ). A representative image of the wound-healing assay (panel $e(i))$ and Matrigel ${ }^{\mathrm{TM}}$ assay (panel $f(i)$ ) is shown. $n=5$. Data is expressed as mean \pm S.E.M. $* p<0.05$ versus vehicle for VEGF 
utilised [23, 24]. RMVEC were exposed to zinc sulphate in the presence and absence of VEGF and sucralose and vasculogenic processes were assessed. The protective effect of sucralose, in attenuating VEGF-induced permeability and vasculogenic processes in RMVEC, was blunted by zinc sulphate (Fig. 3d-h). Interestingly, in the absence of VEGF, zinc sulphate had no impact on endothelial monolayer permeability (Fig. 3c); however, cell proliferation, adhesion, migration, tube formation and angiogenic potential were all significantly increased by zinc sulphate (Fig. 3d-h). These studies indicate that chemical inhibition of T1R2 and T1R3 blocks the protective role of sucralose in the retinal microvascular cell line. To establish the role of each receptor in regulating this protective effect, we next used siRNA to perform molecular inhibition of T1R2 and T1R3. Knockdown of T1R2 or T1R3, confirmed by reduced mRNA and protein expression (Fig. 1), had no impact on RMVEC viability compared to non-specific siRNA control (Fig. 4a). Following knockdown of T1R2, sucralose significantly reduced VEGF-induced permeability of the retinal endothelial barrier, similar to the effect in cells transfected with non-specific siRNA (Fig. 4b (i)). Conversely, following molecular inhibition of T1R3 in RMVEC, sucralose had no effect on VEGF-induced barrier disruption (Fig. 4b (ii)). Likewise, sucralose significantly attenuated VEGF-induced endothelial cell adhesion, migration and tube formation in RMVEC with T1R2, but not T1R3, knockdown (Fig. 4c-e). These data demonstrate that the protective role of sucralose, in blocking VEGFmediated vasculogenic processes in retinal endothelial cells, is mediated through the sweet taste receptor, T1R3.

\section{Sucralose attenuates VEGF-induced vasculogenic processes in the retinal microvascular endothelial cell line by inhibiting Akt activity}

Finally, we sought to understand the mechanism through which T1R3 regulates VEGF-mediated vasculogenic processes in the retinal microvasculature. VEGFR2 is a major regulator of vasculogenesis $[25,26]$ therefore we studied whether sucralose exerted a direct effect on expression of the receptor at the cell surface. As previously described [27], RMVEC exposed to VEGF displayed a significant decrease in VEGFR2 expression at the cell surface (Fig. 5a). Interestingly, VEGFR2 surface expression was not affected by sucralose (Fig. 5a). VEGF-VEGFR2 binding triggers the $\mathrm{PI} 3 \mathrm{~K} /$ Akt signalling pathway which plays a key role in vasculogenic processes therefore we next sought to assess whether sucralose exerts a protective role on the retinal endothelium through Akt signalling $[28,29]$. Activity of Akt1/2,

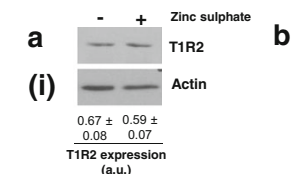

(a.u.)

(ii)

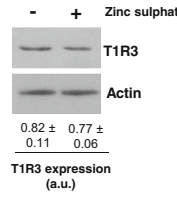

e
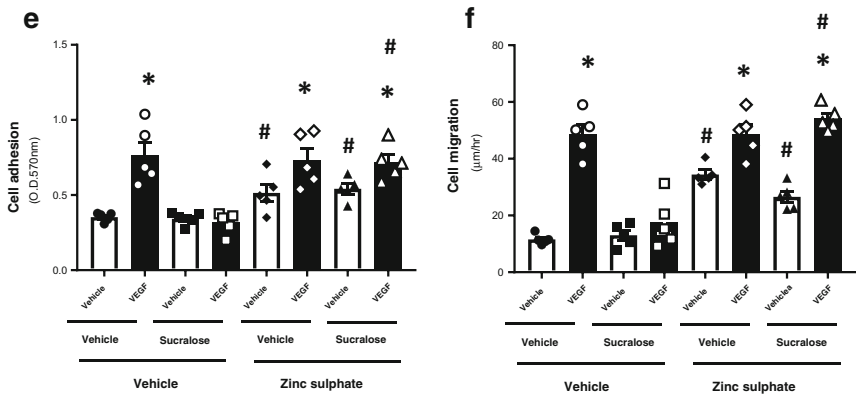

Fig. 3 Inhibition of the sweet taste receptors T1R2 and T1R3, through zinc sulphate, blocks the protective effect of sucralose on VEGF-induced angiogenic processes in the retinal microvascular endothelium. Retinal microvascular endothelial cells were exposed to the sweet taste receptor inhibitor, zinc sulphate $(0.7 \mathrm{mM})$, in the presence and absence of sucralose $(0.1 \mathrm{mM})$ and VEGF $(100 \mathrm{ng} / \mathrm{ml})$. Panel a: protein expression of T1R2 (i) and T1R3 (ii) was assessed by Western blotting of RMVEC lysates. Panel $b$ : cell surface expression of T1R2 (i) and T1R3 (ii) was determined by whole cell ELISA in retinal
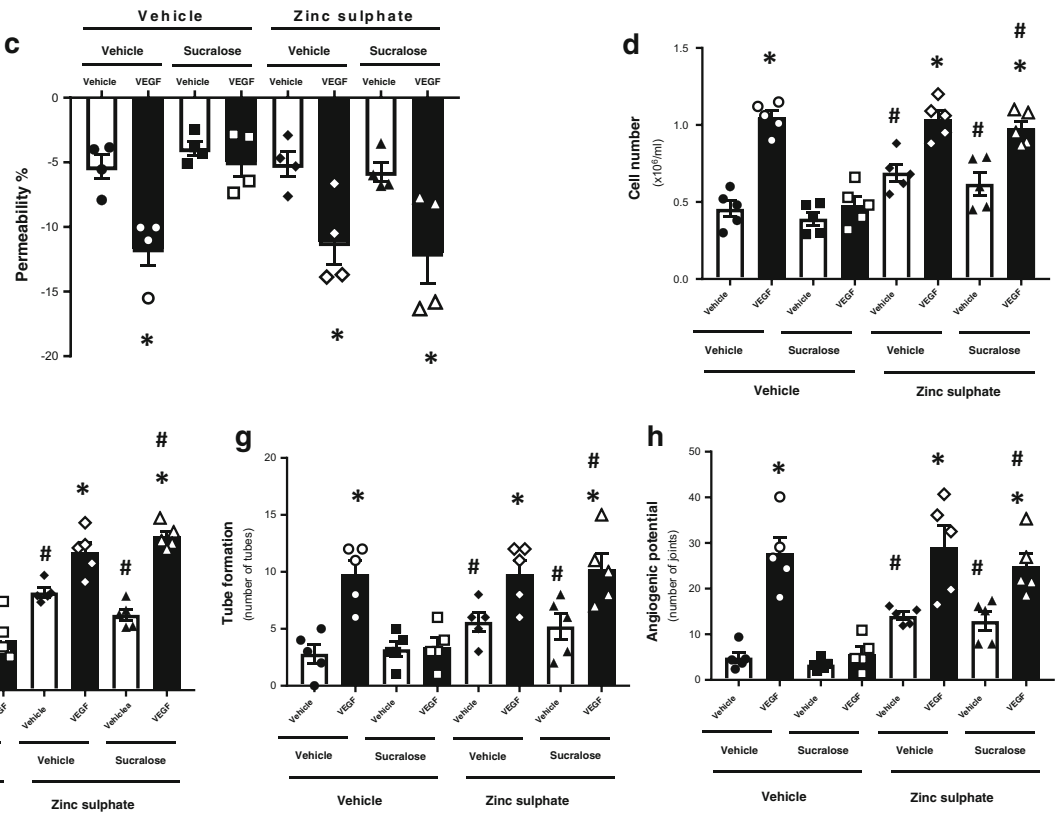

microvascular endothelial cells. Panel $c-h$ : changes in retinal endothelial cell: monolayer permeability (panel c), proliferation (panel $d$ ), adhesion (panel e), migration (panel f), tube formation (panel $g$ ) and angiogenic potential (panel $h$ ) were measured following exposure to sucralose $(0.1 \mathrm{mM})$ in the presence (closed bars) and absence (open bars) of VEGF $(100 \mathrm{ng} / \mathrm{ml}) . n=5$. Data is expressed as mean \pm S.E.M. $* p<0.05$ versus vehicle for VEGF, $\# p<0.05$ versus vehicle for zinc sulphate 


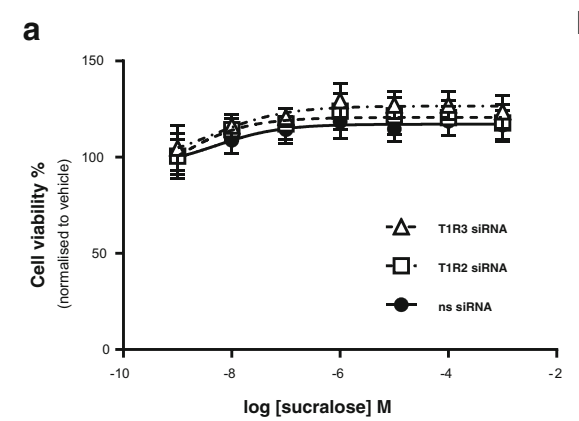

b
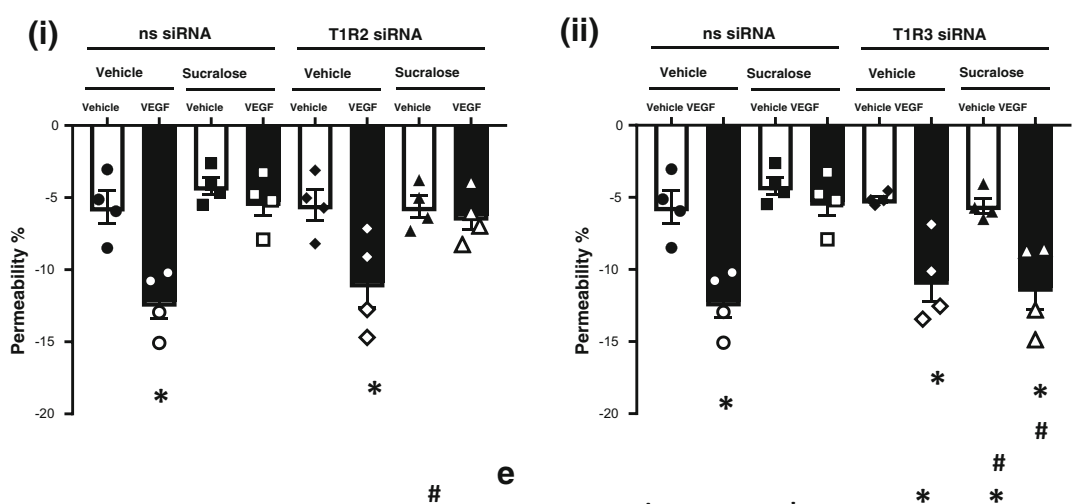

C

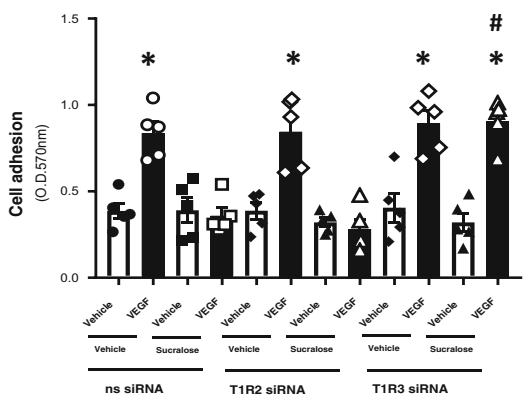

d

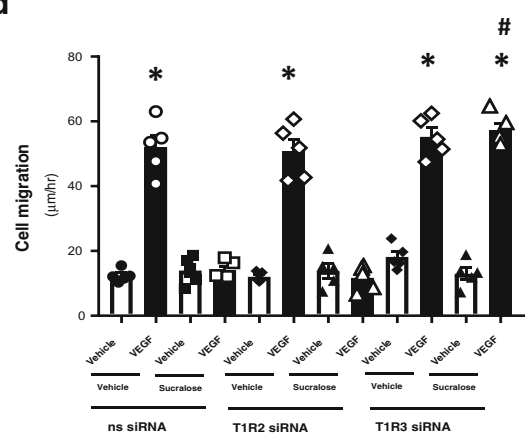

e

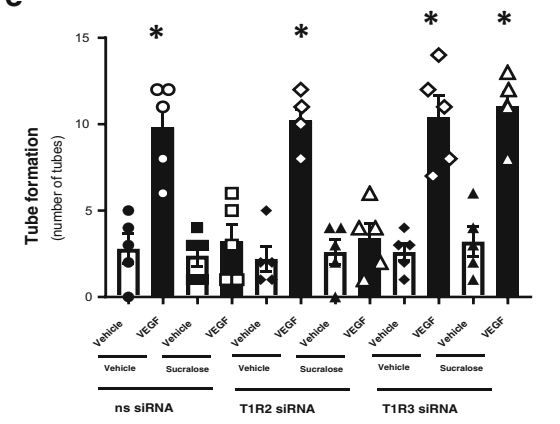

Fig. 4 Inhibition of T1R3, but not T1R2, blocks the protective effect of sucralose on VEGF-induced angiogenesis in the retinal microvascular endothelium. RMVEC were transiently transfected with T1R2 or T1R3 siRNA, or a non-specific scrambled siRNA (ns) for $24 \mathrm{~h}$ prior to treatment with sucralose $(0.1 \mathrm{mM})$ and VEGF $(100 \mathrm{ng} / \mathrm{ml})$. Panel $a$ : cell viability of RMVEC was measured by CCK 8 assay following exposure to sucralose $(1 \mathrm{nM}-1 \mathrm{mM})$. Panel $b$ : changes in retinal endothelial cell monolayer permeability were determined using the
FITC-dextran permeability assay in RMVEC with T1R2 (i) or T1R3 (ii) siRNA knockdown. Panel $c-e$ : changes in retinal endothelial cell: adhesion (panel c), migration (panel $d$ ), tube formation (panel e) were measured following exposure to sucralose $(0.1 \mathrm{mM})$ in the presence (closed bars) and absence (open bars) of VEGF (100 ng/ml). $n=5$. Data is expressed as mean \pm S.E.M. $* p<0.05$ versus vehicle for VEGF, $\# p<0.05$ versus ns siRNA assessed by Western blot analysis of serine 473 [28], was significantly increased in RMVEC exposed to VEGF (Fig. 5b). Interestingly, sucralose had no effect on Akt activity in the absence of VEGF but the sweetener attenuated VEGFinduced Akt phosphorylation (Fig. 5b). To establish whether sucralose protects against VEGF-mediated vasculogenesis through inhibition of Akt, RMVEC were exposed to the Akt activator, SC79, in the presence and absence of VEGF and sucralose. SC79 alone significantly increased cell adhesion, migration and tube formation in retinal endothelial cells (Fig. 5c-e). Following exposure to SC79, VEGF significantly increased permeability (Supplementary Table 1) and vasculogenic processes in RMVEC in the presence of sucralose (Fig. 5c-e). Activation of Akt was thus able to block the protective effect of sucralose and restore VEGF-induced vasculogenesis in the cell model of the retinal endothelium.

Taken together, these date demonstrate that activation of the sweet taste receptor, T1R3, by the artificial sweetener sucralose, protects a retinal microvascular endothelial cell line against VEGF-induced vasculogenesis. We further show that sucralose exerts this protective effect by blocking Akt activation, indicating that the T1R3 represents a novel potential therapeutic target for reducing vascular permeability and vasculogenesis in patients with non-proliferative and proliferative DR.

\section{Discussion}

In this study, we present findings which demonstrate, for the first time, the expression of sweet taste receptors T1R2 and T1R3 in a cell model of the human retinal microvascular endothelium. Our research identifies a role for sweet taste sensing in regulating the retinal endothelium; activation of the sweet taste receptor with sucralose protects the retinal endothelium against VEGF-induced permeability and VEGFinduced vasculogenic processes, linked to non-proliferative and proliferative DR respectively. We further demonstrate that the protective effect of sucralose is mediated through the receptor T1R3, and not T1R2, and by blunting VEGF-induced Akt activity in retinal endothelial cells. Findings from the study demonstrate a novel mechanism through which the retinal endothelium is regulated and indicate a potential therapeutic intervention to reduce the aberrant retinal vasculogenesis observed in patients with DR.

Hyperglycaemia in diabetes is associated with the aberrant vasculogenesis observed in patients with DR [2]. It may therefore appear counter-intuitive for a sweet taste receptor to exert a protective effect on the endothelium; however, glucose concentrations of $\sim 300 \mathrm{mM}$ are needed to stimulate T1R2 and T1R3, concentrations which are not physiological in the 

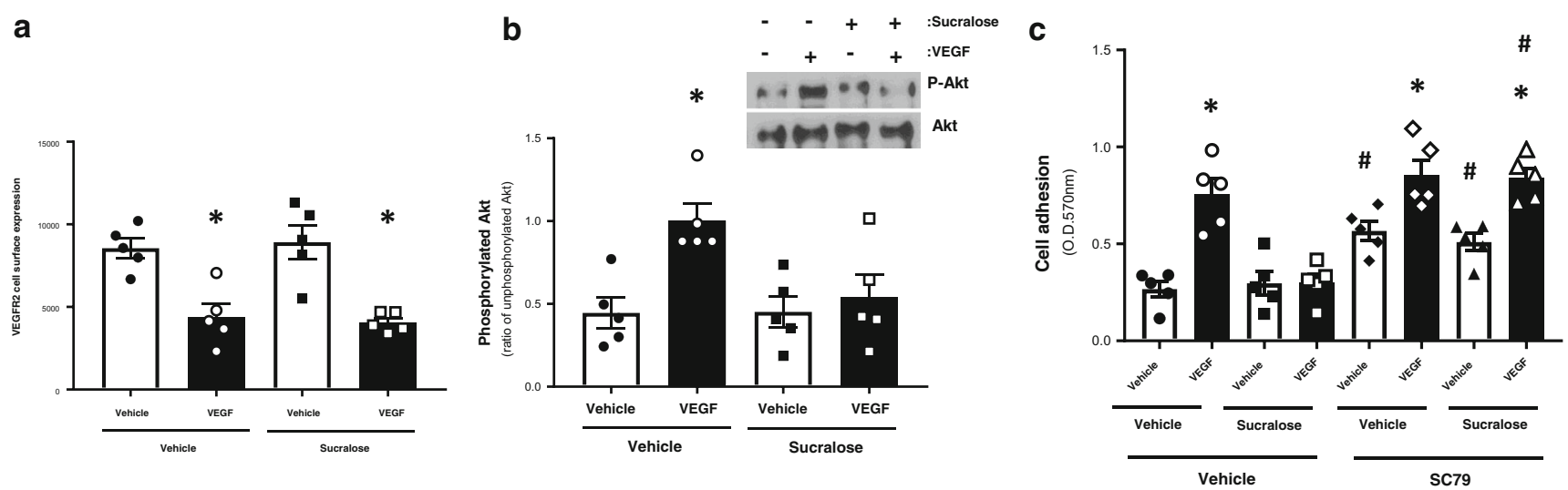

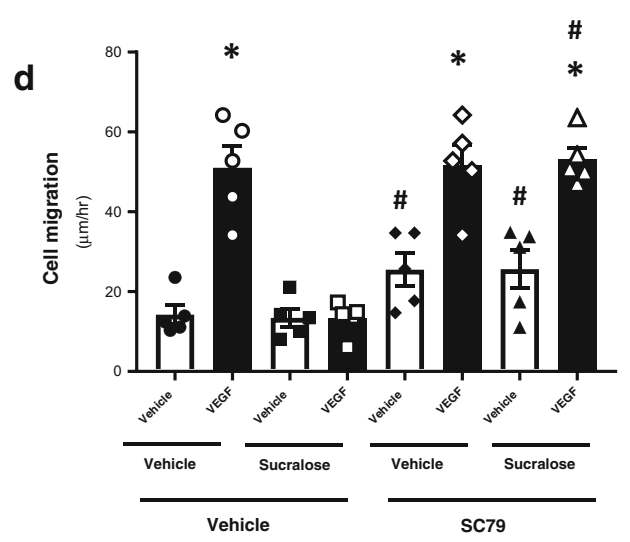

Fig. 5 Sucralose regulates VEGF-induced angiogenesis in the retinal endothelium through suppressing Akt activity. Panel $a$ and $b$ : RMVEC were exposed to sucralose $(0.1 \mathrm{mM})$ in the presence and absence of VEGF (100 ng/ml). VEGFR2 cell surface expression (panel a) and Akt phosphorylation (panel $b$ ) were measured using whole-cell ELISA and Western blotting respectively. A representative blot is shown. Panel $c-e$ : RMVEC were exposed to the Akt activator, SC79 $(10 \mu \mathrm{M})$ or vehicle

circulation [30]. In contrast, sucralose, like many artificial sweeteners, stimulates the sweet taste receptor at low concentrations $(<1 \mathrm{mM})$ [14]. Consumption of artificial sweeteners has increased in the last 15 years with $62 \%$ of all soft drinks consumed being sweetened by these non-nutritive sweeteners [31]. In addition to being recommended for patients with diabetes to reduce intake of sugar, artificial sweeteners are also used as an aid in weight-loss/management. There has, however, been significant controversy in recent years surrounding the use of artificial sweeteners in the diet with both positive and negative impacts on appetite, hunger, weight gain and predisposition for glucose intolerance. As such, the majority of these studies have focused on the small intestine environment, gut microbiota and incretin signalling. These studies suggest increased sweeteners in the diet correlate with weight gain and incidence of diabetes [32]. Following consumption in the diet, a significant proportion of artificial sweetener is absorbed by the small intestine into the systemic circulation and, with the exception of aspartame, is excreted in a largely unmetabolised form [33, 34]. However, there is still a limited understanding of the physiological relevance of T1R2 and T1R3 in the vasculature, and the resulting impact of sweeteners once they are in the circulation.

The blood-retinal barrier forms a selectively-permeable filter maintained by junctional complexes in retinal endothelial cells to form the inner barrier [35]. The pathophysiology of DR is based on multiple components, such as loss of pericyte function and release of inflammatory cytokines by the retinal pigmented epithelium; however, permeability of the retinal endothelial monolayer, via elevated VEGF signalling, is a key pathophysiology of macula edema observed in patients with non-proliferative DR [2, 4]. We have previously demonstrated a role for sweet taste sensing in reducing vascular leak in the lung microvasculature linked to endotoxin-induced pulmonary edema [12]. In the present study, we observe that activation of T1R3, with the artificial sweetener sucralose, exerts a similar effect in protecting the human retinal endothelial monolayer from VEGF-induced permeability. These findings therefore demonstrate a pan-protective role of the receptor which is irrespective of injury stimulus or vascular bed. 
Whilst these studies indicate the protective effect of a T1R3 agonist in a human cell model of the retinal microvasculature, further studies are needed to confirm the physiological relevance of these findings in vivo.

In patients with proliferative DR, aberrant vasculogenic processes such as hyperproliferation and excessive neovascularisation, are observed which are, in part, mediated through pro-angiogenic VEGF signals [5-8]. We and others have previously demonstrated a close association between mechanisms which regulate permeability of the endothelial barrier and vasculogenic processes $[16,19,20]$. Therefore, in the present study, we assessed the effect of T1R3 stimulation on vasculogenic processes in a cell model of the retinal endotheliums, such as proliferation and tube formation. Activation of the sweet taste receptor by sucralose exerted a protective effect on VEGF-mediated vasculogenic processes such as excessive proliferation, adhesion, migration and tube formation. Interestingly, this protection was mediated through the sweet taste receptor T1R3 rather than T1R2. Whilst the sweet taste receptor is typically considered to be a heterodimer of T1R2/T1R3, Nelson et al. have previously demonstrated that T1R3, but not T1R2, can form a homodimer for sweet taste sensing $[13,14]$. It is therefore likely that sucralose binds to the T1R3 homodimer to protect the retinal vasculature against VEGF-induced vasculogenesis. Given that human retinal microvascular endothelial cells express T1R2 mRNA and protein at levels similar to T1R3, yet T1R2 is not involved in permeability or vasculogenesis, this raises interesting questions regarding the potential role for this GPCR in the microvasculature.

Akt has been implicated as a key signalling molecule through which VEGF regulates vasculogenic processes [36]. VEGF-VEGFR2 binding results in PI3K-dependent phosphorylation and activation of Akt resulting in increased endothelial cell proliferation, migration and permeability [37]. Interestingly, artificial sweeteners have been demonstrated to have opposing effects on Akt phosphorylation, depending on the differentiation stage of the cell and the phosphorylation site investigated $[38,39]$. In the present study, we demonstrated that sucralose blocked VEGF-induced phosphorylation of Akt at $\mathrm{Ser}^{473}$. Given the role of Akt phosphorylation at this residue in regulating FOXO1 target genes associated with vasculogenesis [40, 41], our data indicates that T1R3 activation blocks vasculogenic processes through Akt dephosphorylation. Indeed, we observed that chemical activation of Akt reversed the protective effects of sucralose on vasculogenesis. Sucralose had no impact on Akt phosphorylation, or vasculogenic processes, in the healthy endothelium (in the absence of VEGF). Therefore it is possible that T1R3 signalling downregulates a specific kinase which is upregulated by VEGF signalling, such as mTOR [37]; however, further studies are needed to fully understand the molecular mechanism through which T1R3 regulates Akt activity in the retinal endothelium.
At present, treatment for patients with non-proliferative and proliferative DR is based on intravitreous injection of anti-VEGF agents, such as ranibizumab, and panretinal laser photocoagulation therapy [42]. Whilst these treatments are effective in reducing retinopathy in patients, long-term use of anti-VEGF agents has been linked to eye inflammation and capillary regression of the remaining healthy microvasculature [43] Given the increasing number of patient with diabetes, and the high chance of these patients developing retinopathy $[1,2]$, there is a significant need to consider alternate potential therapeutic approaches to reduce permeability and vasculogenesis in the retinal microvasculature. In the present study, we demonstrate that sucralose blocks VEGF-induced permeability and vasculogenic processes. Interestingly, in the healthy endothelium, in the absence of VEGF, we demonstrate that sucralose has no effect on permeability or vasculogenesis. Our studies therefore indicate that T1R3 represents a novel therapeutic target with potential to reduce permeability and vasculogenesis in the retinal endothelium in patients with both proliferative and nonproliferative DR. Further research is needed to understand the physiological effect of sweeteners on pathogenic events leading to retinopathy, as well as the potential side effects of sweeteners in the vasculature, similar to those seen with current anti-VEGF treatment.

Author contributions P. Lizunkova, E. Enuwosa and H. Chichger designed and performed the experiments and analysed data. H. Chichger interpreted results, conceived and designed the overall study, reviewed the data, and wrote the manuscript. H. Chichger is the guarantor of this work and, as such, had full access to all the data in the study and takes responsibility for the integrity of the data and the accuracy of the data analysis.

Funding This material is based on work supported by Diabetes UK Grant 15/0005284 (H. Chichger), American Heart Association Grant 13POST16860031 (H. Chichger) and Wellcome Trust Vacation Studentship 202624/Z/16/Z (P. Lizunkova and H. Chichger). The sponsor had no role in the design or conduct of this research.

\section{Compliance with ethical standards}

Conflict of interest The authors declare that they have no conflict of interest.

Ethical considerations All experimental protocols were approved by the Departmental Research Ethics Panel at Anglia Ruskin University prior to start. This article does not contain any studies with human participants or animals performed by any of the authors.

Open Access This article is distributed under the terms of the Creative Commons Attribution 4.0 International License (http:// creativecommons.org/licenses/by/4.0/), which permits unrestricted use, distribution, and reproduction in any medium, provided you give appropriate credit to the original author(s) and the source, provide a link to the Creative Commons license, and indicate if changes were made. 


\section{References}

1. Ogurtsova K, da Rocha Fernandes JD, Huang Y et al (2017) IDF diabetes atlas: global estimates for the prevalence of diabetes for 2015 and 2040. Diabetes Res Clin Pract 128:40-50

2. Wong TY, Cheung CM, Larsen M et al (2016) Diabetic retinopathy. Nat Rev Dis Primers 2:16012. https://doi.org/10.1038/ nrdp.2016.12

3. Mohamed Q, Gillies MC, Wong TY (2007) Management of diabetic retinopathy: a systematic review. JAMA 298(8):902-916

4. Frank RN (2004) Diabetic retinopathy. N Engl J Med 350(1):4858. https://doi.org/10.1056/NEJMra021678

5. Aiello LP, Avery RL, Arrigg PG et al (1994) Vascular endothelial growth factor in ocular fluid of patients with diabetic retinopathy and other retinal disorders. N Engl J Med 331(22):1480-1487. https://doi.org/10.1056/NEJM199412013312203

6. Simo R, Carrasco E, Garcia-Ramirez M et al (2006) Angiogenic and antiangiogenic factors in proliferative diabetic retinopathy. Curr Diabetes Rev 2(1):71-98

7. Zehetner C, Kirchmair R, Kralinger M et al (2013) Correlation of vascular endothelial growth factor plasma levels and glycemic control in patients with diabetic retinopathy. Acta Ophthalmol 91(6): 470. https://doi.org/10.1111/aos.12081

8. Ozturk BT, Bozkurt B, Kerimoglu H et al (2009) Effect of serum cytokines and VEGF levels on diabetic retinopathy and macular thickness. Mol Vis 15:1906-1914

9. Ferrara N (2004) Vascular endothelial growth factor: basic science and clinical progress. Endocr Rev 25(4):581-611. https://doi.org/ 10.1210/er.2003-0027

10. Simo R, Sundstrom JM, Antonetti DA (2014) Ocular anti-VEGF therapy for diabetic retinopathy: the role of VEGF in the pathogenesis of diabetic retinopathy. Diabetes Care 37(4):893-899. https:// doi.org/10.2337/dc13-2002

11. Lim LS, Cheung CMG, Mitchell $P$ et al (2011) Emerging evidence concerning systemic safety of anti-VEGF agents-should ophthalmologists be concerned? Am J Ophthalmol 152(3):329-331

12. Harrington EO, Vang A, Braza J et al (2018) Activation of the sweet taste receptor, T1R3, by the artificial sweetener sucralose regulates the pulmonary endothelium. Am J Physiol Lung Cell Mol Physiol 314(1):176. https://doi.org/10.1152/ajplung.00490.2016

13. Nelson G, Hoon MA, Chandrashekar J et al (2001) Mammalian sweet taste receptors. Cell 106(3):381-390

14. Li X, Staszewski L, Xu H et al (2002) Human receptors for sweet and umami taste. Proc Natl Acad Sci U S A 99(7):4692-4696. https://doi.org/10.1073/pnas.072090199

15. Abraham S, Yeo M, Montero-Balaguer M et al (2009) VECadherin-mediated cell-cell interaction suppresses sprouting via signaling to MLC2 phosphorylation. Curr Biol 19(8):668-674. https://doi.org/10.1016/j.cub.2009.02.057

16. Yoshioka K, Yoshida K, Cui H et al (2012) Endothelial PI3KC2alpha, a class II PI3K, has an essential role in angiogenesis and vascular barrier function. Nat Med 18(10):1560-1569. https://doi. org/10.1038/nm.2928

17. Im E, Kazlauskas A (2007) Src family kinases promote vessel stability by antagonizing the rho/ROCK pathway. J Biol Chem 282(40):29122-29129

18. Bueter M, Miras AD, Chichger $\mathrm{H}$ et al (2011) Alterations of sucrose preference after roux-en-Y gastric bypass. Physiol Behav 104(5): 709-721. https://doi.org/10.1016/j.physbeh.2011.07.025

19. Chichger H, Duong H, Braza J et al (2015) p18, a novel adaptor protein, regulates pulmonary endothelial barrier function via enhanced endocytic recycling of VE-cadherin. FASEB J 29(3):868881. https://doi.org/10.1096/fj.14-257212

20. Chichger H, Braza J, Duong H et al (2015) Neovascularization in the pulmonary endothelium is regulated by the endosome: Rab4- mediated trafficking and p18-dependent signaling. Am J Physiol Lung Cell Mol Physiol 309(7):L700-L709. https://doi.org/10. 1152/ajplung.00235.2015

21. Masuda K, Koizumi A, Nakajima K et al (2012) Characterization of the modes of binding between human sweet taste receptor and lowmolecular-weight sweet compounds. PLoS One 7(4):e35380. https://doi.org/10.1371/journal.pone.0035380

22. Cui J, Gong R, Hu S et al (2018) Gambogic acid ameliorates diabetes-induced proliferative retinopathy through inhibition of the HIF-1alpha/VEGF expression via targeting PI3K/AKT pathway. Life Sci 192:293-303

23. Elliott RA, Kapoor S, Tincello DG (2011) Expression and distribution of the sweet taste receptor isoforms T1R2 and T1R3 in human and rat bladders. J Urol 186(6):2455-2462. https://doi.org/10.1016/ j.juro.2011.07.083

24. Keast RS, Canty TM, Breslin PA (2004) Oral zinc sulfate solutions inhibit sweet taste perception. Chem Senses 29(6):513-521. https:// doi.org/10.1093/chemse/bjh053

25. Herbert SP, Stainier DY (2011) Molecular control of endothelial cell behaviour during blood vessel morphogenesis. Nat Rev Mol Cell Biol 12(9):551-564. https://doi.org/10.1038/ nrm3176

26. Quinn TP, Peters KG, Vries CD et al (1993) Fetal liver kinase 1 is a receptor for vascular endothelial growth factor and is selectively expressed in vascular endothelium. Proc Natl Acad Sci U S A 90(16):7533-7537

27. Basagiannis D, Zografou S, Murphy C et al (2016) VEGF induces signalling and angiogenesis by directing VEGFR2 internalisation through macropinocytosis. J Cell Sci 129(21): 4091-4104

28. Chen J, Somanath PR, Razorenova O et al (2005) Akt1 regulates pathological angiogenesis, vascular maturation and permeability in vivo. Nat Med 11(11):1188-1196

29. Ruan GX, Kazlauskas A (2013) Lactate engages receptor tyrosine kinases Axl, Tie2, and vascular endothelial growth factor receptor 2 to activate phosphoinositide 3-kinase/Akt and promote angiogenesis. J Biol Chem 288(29):21161-21172. https://doi.org/10.1074/ jbc.M113.474619

30. Mace OJ, Affleck J, Patel N et al (2007) Sweet taste receptors in rat small intestine stimulate glucose absorption through apical GLUT2. J Physiol Lond 582(Pt 1):379-392

31. Gardner C, Wylie-Rosett J, Gidding SS et al (2012) Nonnutritive sweeteners: current use and health perspectives: a scientific statement from the American Heart Association and the American Diabetes Association. Circulation 126(4):509-519

32. Suez J, Korem T, Zeevi D et al (2014) Artificial sweeteners induce glucose intolerance by altering the gut microbiota. Nature 514(7521):181-186. https://doi.org/10.1038/nature13793

33. Roberts A, Renwick AG, Sims J et al (2000) Sucralose metabolism and pharmacokinetics in man. Food Chem Toxicol 38(Suppl 2):31

34. Sweatman TW, Renwick AG, Burgess CD (1981) The pharmacokinetics of saccharin in man. Xenobiotica 11(8):531-540

35. Shakib M, Cunha-Vaz JG (1966) Studies on the permeability of the blood-retinal barrier. IV Junctional complexes of the retinal vessels and their role in the permeability of the blood-retinal barrier. Exp Eye Res 5(3):229-234

36. Shiojima I, Walsh K (2002) Role of Akt signaling in vascular homeostasis and angiogenesis. Circ Res 90(12):1243-1250

37. Holmes K, Roberts OL, Thomas AM et al (2007) Vascular endothelial growth factor receptor-2: structure, function, intracellular signalling and therapeutic inhibition. Cell Signal 19(10):2003-2012

38. Masubuchi Y, Nakagawa Y, Medina J et al (2017) T1R3 homomeric sweet taste receptor regulates adipogenesis through Galphasmediated microtubules disassembly and Rho activation in 3T3-L1 
cells. PLoS One 12(5):e0176841. https://doi.org/10.1371/journal. pone. 0176841

39. Simon BR, Parlee SD, Learman BS et al (2013) Artificial sweeteners stimulate adipogenesis and suppress lipolysis independently of sweet taste receptors. J Biol Chem 288(45):32475-32489. https://doi.org/10.1074/jbc.M113.514034

40. Daly C, Wong V, Burova E et al (2004) Angiopoietin-1 modulates endothelial cell function and gene expression via the transcription factor FKHR (FOXO1). Genes Dev 18(9):1060-1071. https://doi. org/10.1101/gad.1189704

41. Guertin DA, Stevens DM, Thoreen CC et al (2006) Ablation in mice of the mTORC components raptor, rictor, or mLST8 reveals that mTORC2 is required for signaling to Akt-FOXO and PKCalpha, but not S6K1. Dev Cell 11(6):859-871

42. Network,Writing Committee for the Diabetic Retinopathy Clinical Research, Gross JG, Glassman AR et al (2015) Panretinal photocoagulation vs intravitreous ranibizumab for proliferative diabetic retinopathy: a randomized clinical trial. JAMA 314(20):2137-2146. https://doi.org/10.1001/jama.2015.15217

43. Saint-Geniez M, Kurihara T, Sekiyama E et al (2009) An essential role for RPE-derived soluble VEGF in the maintenance of the choriocapillaris. Proc Natl Acad Sci U S A 106(44):1875118756. https://doi.org/10.1073/pnas.0905010106 